\title{
Amathonte : les abords Sud-Ouest de l'agora - 2016
}

Ludovic Thély, Julien Adam, Dominique Barcat, Camille Castres, FrançoisDominique Deltenre, Antigone Marangou et Cécile Rocheron

\section{OpenEdition Journals}

Édition électronique

URL : http://journals.openedition.org/baefe/1281

DOI : $10.4000 /$ baefe. 1281

ISSN : 2732-687X

Éditeur

ResEFE

\section{Référence électronique}

Ludovic Thély, Julien Adam, Dominique Barcat, Camille Castres, François-Dominique Deltenre,

Antigone Marangou et Cécile Rocheron, «Amathonte : les abords Sud-Ouest de l'agora - 2016 » [notice archéologique], Bulletin archéologique des Écoles françaises à l'étranger [En ligne], Chypre, mis en ligne le 26 novembre 2020, consulté le 16 décembre 2020. URL : http://journals.openedition.org/ baefe/1281 ; DOI : https://doi.org/10.4000/baefe.1281

Ce document a été généré automatiquement le 16 décembre 2020.

\section{cc) (i) (3)}

Le Bulletin archéologique des Écoles françaises à l'étranger est mise à disposition selon les termes de la Licence Creative Commons Attribution - Pas d'Utilisation Commerciale - Pas de Modification 4.0 International. 


\title{
Amathonte : les abords Sud-Ouest de l'agora - 2016
}

\author{
Ludovic Thély, Julien Adam, Dominique Barcat, Camille Castres, François- \\ Dominique Deltenre, Antigone Marangou et Cécile Rocheron
}

\section{NOTE DE L'AUTEUR}

Autorité nationale présente : Yiannis Violaris (Département des Antiquités de Chypre, District de Limassol)

Composition de l'équipe de terrain : Ludovic Thély, responsable d'opération ; Julien Adam et François-Dominique Deltenre, chefs de secteur ; Camille Castres, architecte ; Antigone Marangou et Cécile Rocheron, céramologues. Ont en outre participé aux opérations quatorze étudiants français, belges, italiens et chypriotes.

Partenariats institutionnels : École française d'Athènes, Ministère de l'Europe et des Affaires étrangères.

Établissements porteurs de l'opération : École française d'Athènes.

Remerciements : La mission exprime sa reconnaissance à Marina Solomidou-Ieronymidou, directrice du Département des Antiquités de Chypre, et à Yiannis Violaris, archéologue responsable du district de Limassol, pour leur collaboration et confiance.

Données scientifiques produites :

Mission française d'Amathonte par l'EFA

Mission française d'Amathonte par l'Ambassade de France à Chypre

\section{Introduction}

1 La campagne de fouilles menée depuis l'automne 2014 répond à un programme conjoint de l'École française d'Athènes et du Ministère des Affaires étrangères avec l'appui précieux du Département des Antiquités de Chypre. L'objectif de l'année 2016, après la 
campagne fructueuse de l'an précédent, consistait à confirmer ou d'infirmer, dans une logique d'interdisciplinarité ${ }^{1}$ l'hypothèse de l'existence d'un port intérieur à Amathonte qui aurait été en eau aux époques archaïque et classique, puis aurait fonctionné comme une base arrière du grand port de guerre hellénistique fouillé par J.Y. Empereur au milieu des années $1980^{2}$. Les principaux acquis obtenus en 2014 puis en 2015, dont nous rappelons ci-dessous les grands traits, ont conduit à l'organisation d'une troisième campagne archéologique du 29 août au 23 septembre 2016, dans le cadre d'un programme quadriennal décidé en accord avec les responsables chypriotes. Parmi les principaux résultats, signalons d'emblée : 1) que la limite des constructions de l'agora et des quartiers périphériques, qui semblait avoir été atteinte au Sud en 2014, doit être légèrement repoussée au vu de la découverte de structures nouvelles; 2) qu'un imposant bâtiment dont la fonction nous échappe encore est toujours en cours de dégagement ; 3) que, sans doute pour la première fois, nous sommes en mesure d'établir un phasage chronologique précis de l'occupation de cette partie du site de la fin de l'époque hellénistique à la période protobyzantine, incluant une documentation claire sur certaines traces d'activités tardives jusqu'alors méconnues à Amathonte.

\section{Campagnes 2014-2015 : rappel des résultats}

2 Les fouilles conduites dans la zone du bassin intérieur en 2014 s'appuyaient sur une double étude préliminaire effectuée au printemps: une série de neuf carottages (Antoine Chabrol) attestaient une évolution du paysage antique en trois temps (paléoplage, comblement lagunaire et colluvionnement sédimentaire) ; une prospection géophysique (C. Benech) rendait compte d'une zone de forte résistivité au Sud-Ouest de l'agora, correspondant sans doute à la présence d'un bâtiment ou d'une structure de grande taille ${ }^{3}$.

Les sondages ouverts à l'automne 2014 ont mis en évidence la présence de deux murs parallèles (Sect. 01/N), d'une largeur maximale de 1,80 m (fig. 1). L'UC 1001, la plus au Nord, présente un double parement avec remplissage de terre et de moellons. L'UC 1003 était d'interprétation plus délicate, d'autant que la largeur de l'espace entre les deux structures ne dépasse pas 1,40 m et est traversée par une canalisation (UC 1004) audessus de laquelle un niveau de circulation peut être identifié (US 1017 et 1021), sur lequel reposait une série d'une quinzaine d'amphores complètes écrasées sous l'effondrement de plusieurs blocs appartenant sans doute à la structure UC 1001 (fig. 2). Le niveau de fondation des UC 1001 et 1003 paraissait atteint à la fin de la campagne 2014 (fig. 3): le matériel, étudié au printemps 2015, a révélé une homogénéité de la couche (sans pour autant que l'on ait clairement trouvé la tranchée de fondation), dont le terminus post quem peut être daté du dernier tiers du $\mathrm{I}^{\mathrm{er}} \mathrm{s}$. de n. è (US 1030). Le matériel amphorique (US 1017) se place quant à lui entre le $\mathrm{II}^{\mathrm{e}}$ et le $\mathrm{I}^{\mathrm{er}} \mathrm{s}$. av. n. è. 
Fig. 1. Structures (UC 1001, 1002, 1003 et 1004) dégagées à la fin de la fouille 2014 vues du Nord.

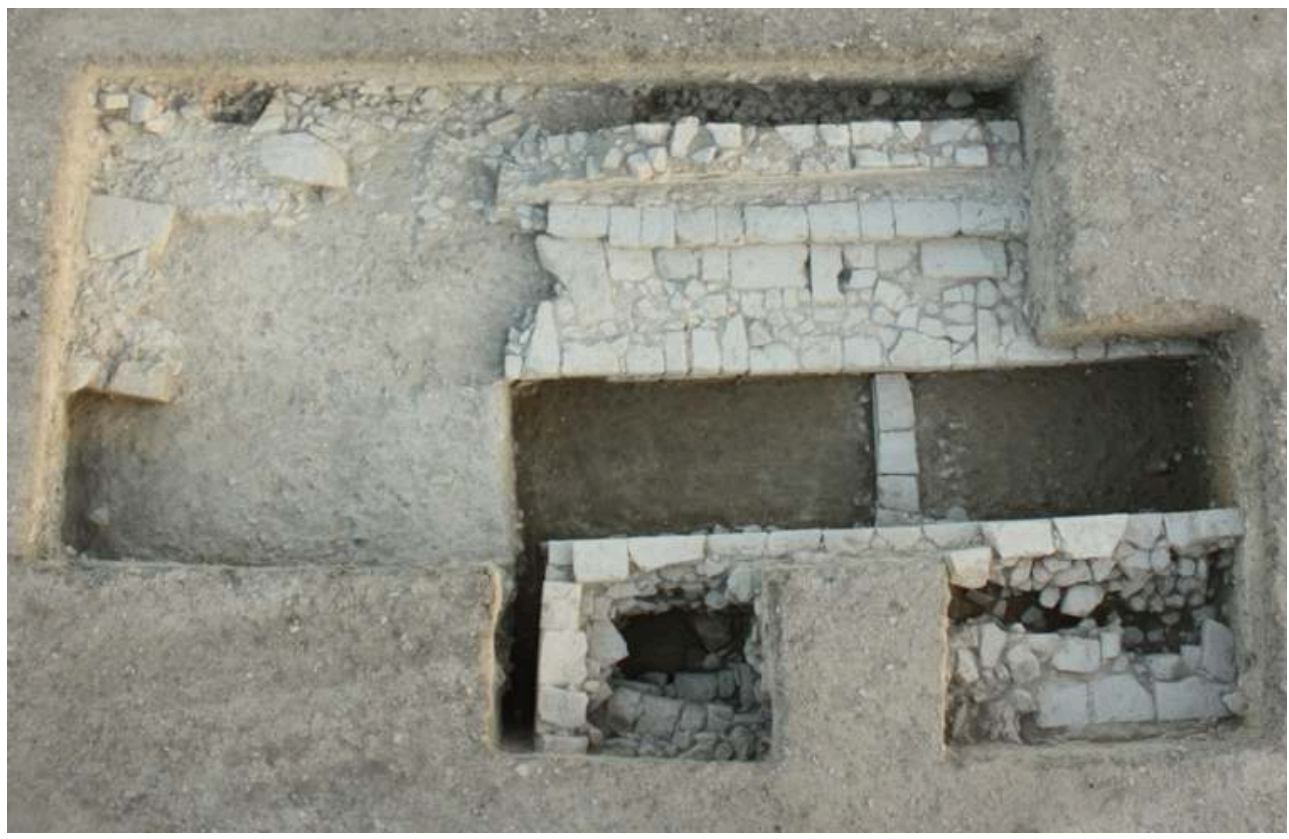

L. Thély / EFA.

Fig. 2. US 1017.

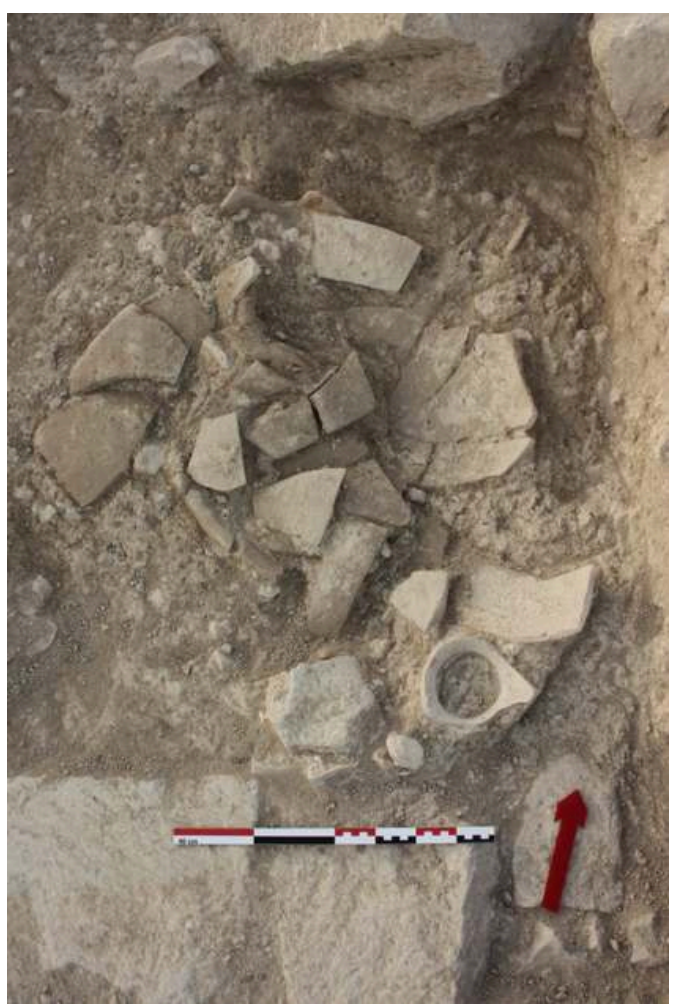

L. Thély / EFA. 
Fig. 3. Niveau de fondation des structures.

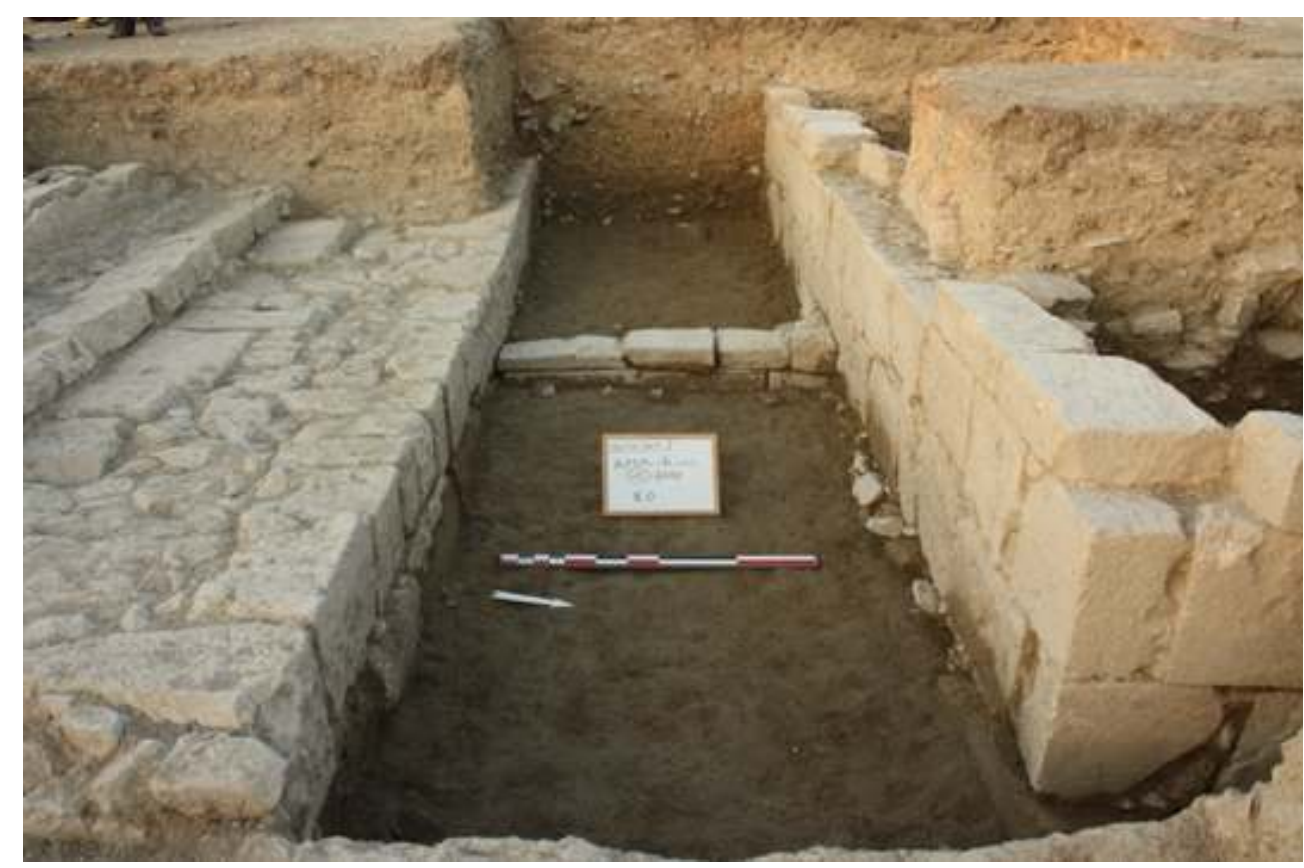

L. Thély / EFA.

4 La campagne 2015 partait donc de résultats encourageants obtenus à la suite de la première fouille et de la post-fouille qui s'en est suivie.

5 La question du phasage chronologique est malgré tout rendue délicate par l'état actuel des conclusions de l'étude céramique. En effet, la presque totalité des couches décrites supra sont datées de la fin de l'époque hellénistique et du $\mathrm{I}^{\mathrm{er}} \mathrm{s}$. de $\mathrm{n}$. è. Les résultats sont toutefois conditionnés par deux facteurs: d'une part, les datations proposées se fondent essentiellement sur la céramique sigillée et les amphores; d'autre part, un certain nombre de couches contiennent trop peu de matériel datant. On distingue malgré tout deux phases d'occupation et d'activité du site: la première est contemporaine et directement liée aux structures massives dégagées par la fouille; la seconde leur est postérieure, à une époque où les principaux murs avaient déjà été remblayés.

6 Les US les plus récentes présentent plusieurs caractéristiques indiquant une occupation de l'espace destinée à une activité artisanale : le creusement de deux fosses et leur remplissage, notamment par des résidus du travail de la pourpre (seule utilité connue des murex que contenait l'une des fosses), l'abondance d'un type rare de céramique culinaire à fond annulaire présentant des traces de pigments, les zones de déchets de taille et la présence d'une terre rubéfiée dont la couleur tire du rouge au jaune vif, portent à croire que, lorsque les structures furent remblayées (sans doute au cours du $\mathrm{I}^{\mathrm{er}}$ ou $\mathrm{II}^{\mathrm{e}} \mathrm{s}$. de n. è.), la zone a servi de lieu de rejet à une activité de teinturerie. Il n'y aurait là rien de surprenant au vu de la topographie générale du secteur, suffisamment à l'écart de l'agora et proche de la mer. L'alignement des deux fosses du Sect. 02, le long d'une diagonale Nord-Est / Sud-Ouest, pourrait indiquer qu'elles ont été creusées de manière très rapprochée dans le temps. On ignore à ce stade si elles avaient une fonction précise, autre que celle de dépotoir de matériel. Une autre activité qui précède ces fosses semble également s'être déployée, comme paraissent l'indiquer à la fois 
l'important foyer (US 2010) et son contenu. Elle pourrait impliquer une activité métallurgique légère, mais aussi un travail des pigments.

7 La seconde phase que l'on peut identifier en chronologie relative est celle qui s'appuie et fonctionne concomitamment avec l'UC 1001 et peut-être également l'UC 1003. Il semble que c'est à l'époque augustéenne ou tibérienne qu'un réaménagement et une monumentalisation de la ville basse d'Amathonte furent entrepris. La construction des deux importantes structures, exactement parallèles, aux abords Sud-Ouest de l'agora, doit se placer dans ce cadre chronologique: l'autel en l'honneur de Ptolémée $\mathrm{X}$ (AM 3726) donne en outre un premier repère chronologique fiable ${ }^{4}$. La difficulté vient surtout de leur arasement et de leur abandon: l'étude du matériel céramique semble indiquer que ces opérations ont pu intervenir bien avant le $\mathrm{III}^{\mathrm{e}} \mathrm{s}$. de n. è., peut-être même une centaine d'années au plus après la construction de l'ensemble. L'UC 1002 marque la limite méridionale des structures. L'étude de la stratigraphie a d'autre part montré que, en dépit de son aspect bien différent des autres constructions, il fut remblayé à la même époque que l'UC 1003 auquel il est accolé. Il était manifestement destiné à avoir une assez faible hauteur afin de permettre le support d'un drain à ciel ouvert et devait être enduit pour le passage de l'eau.

8 La fouille des deux secteurs a apporté plusieurs éléments de réponse concernant l'organisation spatiale de la ville basse d'Amathonte dans la zone supposée du bassin intérieur. À l'époque où les structures furent édifiées, à la toute fin de l'époque hellénistique ou au début du Principat, l'anse naturelle était désormais comblée, comme le suggère les résultats de l'enquête géomorphologique. Si les voies et les espaces de communication entre le front de mer et l'agora étaient assurément hors d'eau, la zone demeurait malgré tout marécageuse, ce qui conduit à l'interprétation, sensée mais non prouvée définitivement, de l'utilisation des amphores complètes découvertes au-dessus des fondations du mur Nord (US 1017) comme moyen d'étanchéiser les niveaux de circulation. Le colluvionnement naturel de la zone a ainsi sans doute permis le réaménagement des espaces proches de l'agora. La question de la limite entre les espaces monumentaux aménagés (agora, quartiers d'habitation tardifs) et le comblement naturel du bassin a, selon toute vraisemblance, été éclaircie : les données de la prospection géophysique confirment en outre les résultats obtenus par la fouille. Aucune structure antérieure $\mathrm{au} \mathrm{II}^{\mathrm{e}} \mathrm{s}$. de n. è. n'a été révélée au-delà du mur le plus méridional. La stratigraphie, très homogène à cet endroit, suggère par ailleurs un comblement uniforme de la zone.

9 Malheureusement, les structures mises au jour lors des deux premières campagnes ne peuvent pas être encore identifiées. Cette lacune est bien sûr due à l'absence d'un matériel suffisamment significatif, témoignage d'un édifice précis et de son organisation intérieure. Il convient de noter toutefois l'importante superficie sur laquelle les murs se déploient, ainsi que l'arrêt brutal du retour Nord de l'UC 1001 : il serait tentant d'y voir un dispositif de murs d'enceinte, non pas nécessairement à vocation défensive, mais peut-être tout simplement civique, aménagée au Nord-Est par une entrée monumentale. Les autres structures (UC 1002, 1003 et 1004) constituent encore pour l'heure un épineux problème : on peine en effet à établir le lien fonctionnel entre elles et l'antériorité ou la contemporanéité des unes par rapport aux autres. 


\section{Nouvelles observations stratigraphiques} prévoyait l'extension de la zone fouillée à l'Ouest, sans laisser de berme dans le secteur 02 , et en conservant un espace de circulation et d'étude stratigraphique en coupe d' $1 \mathrm{~m}$ de large dans le premier secteur.

Dans ce cas précis, les objectifs étaient triple: retrouver les fondations des deux structures parallèles, UC 1001 et UC 1003, notamment dans la zone du sondage C, mais également à travers une extension prévue à l'Ouest; poursuivre la fouille de la zone Sud (sondage B) afin de vérifier l'exactitude des informations obtenues en 2015 ; ouvrir un sondage contigu au sondage $\mathrm{C}$, en direction du Sud, à la demande des autorités chypriotes afin de s'assurer de l'absence, à ce moment de la fouille, de structures portuaires enfouies.

12 La connexion physique directe entre les deux «zones» du Sect. 02 avait pour but d'établir des équivalences stratigraphiques entre elles et de comprendre de manière plus extensive l'espace septentrional de la fouille. Le carré fouillé en 2016 ( 7 x 7 m) est donc parfaitement contigu à celui de 2015, sauf au Nord où l'on a préféré suivre la limite créée par l'opération de nettoyage et de préparation du Sect.02 effectuée en 2015. L'objectif principal était de comprendre la structure apparue lors de l'opération de nettoyage et de préparation du Sect. 02 en 2015 (UC 2005) qui présentait un fil de blocs taillés et des traces de mortier sur les pierres de l'élévation.

13 La fouille s'est d'abord concentrée sur les couches supérieures qui subsistaient seulement dans la partie Nord-Ouest du sondage avant de procéder à l'enlèvement progressif des couches sous-jacentes situées sur le reste de la surface qui était l'objet de l'opération en 2016. À l'issue de cette campagne, plusieurs éléments interprétatifs apparaissent. Sur base de la séquence stratigraphique, nous sommes en mesure de proposer un premier phasage global du secteur qui éclaire les dynamiques d'occupation de la zone (fig. 4-5). 
Fig. 4. Diagramme de Harris du Secteur 02.

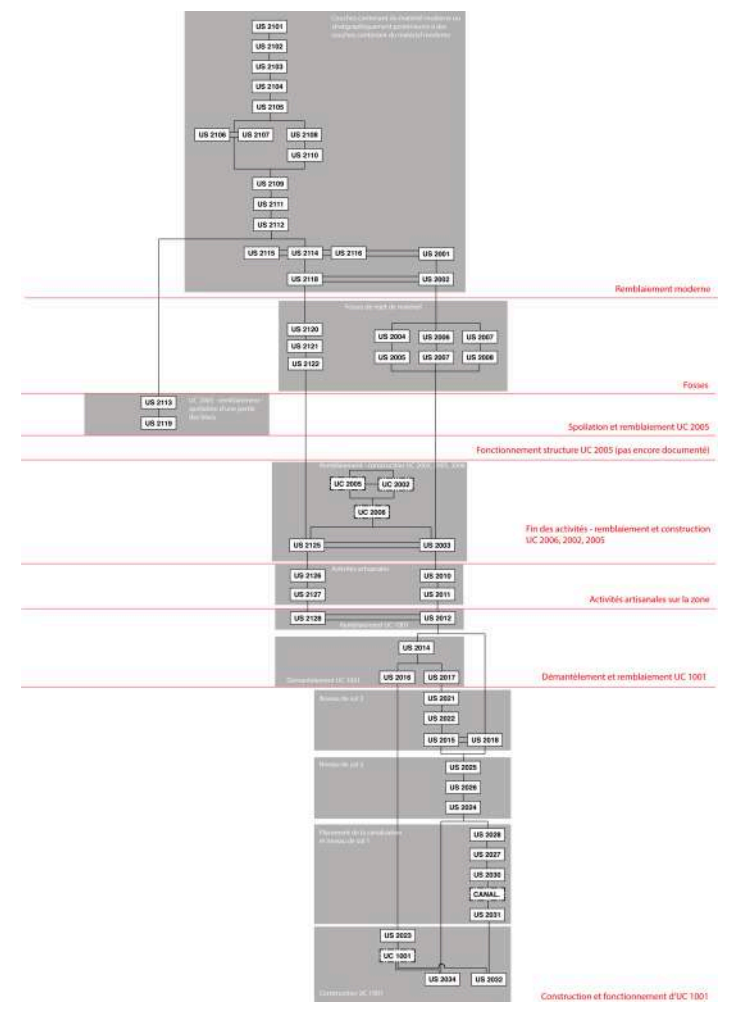

L. Thély / EFA.

Fig. 5. Vue des structures à la fin de la campagne 2016 .

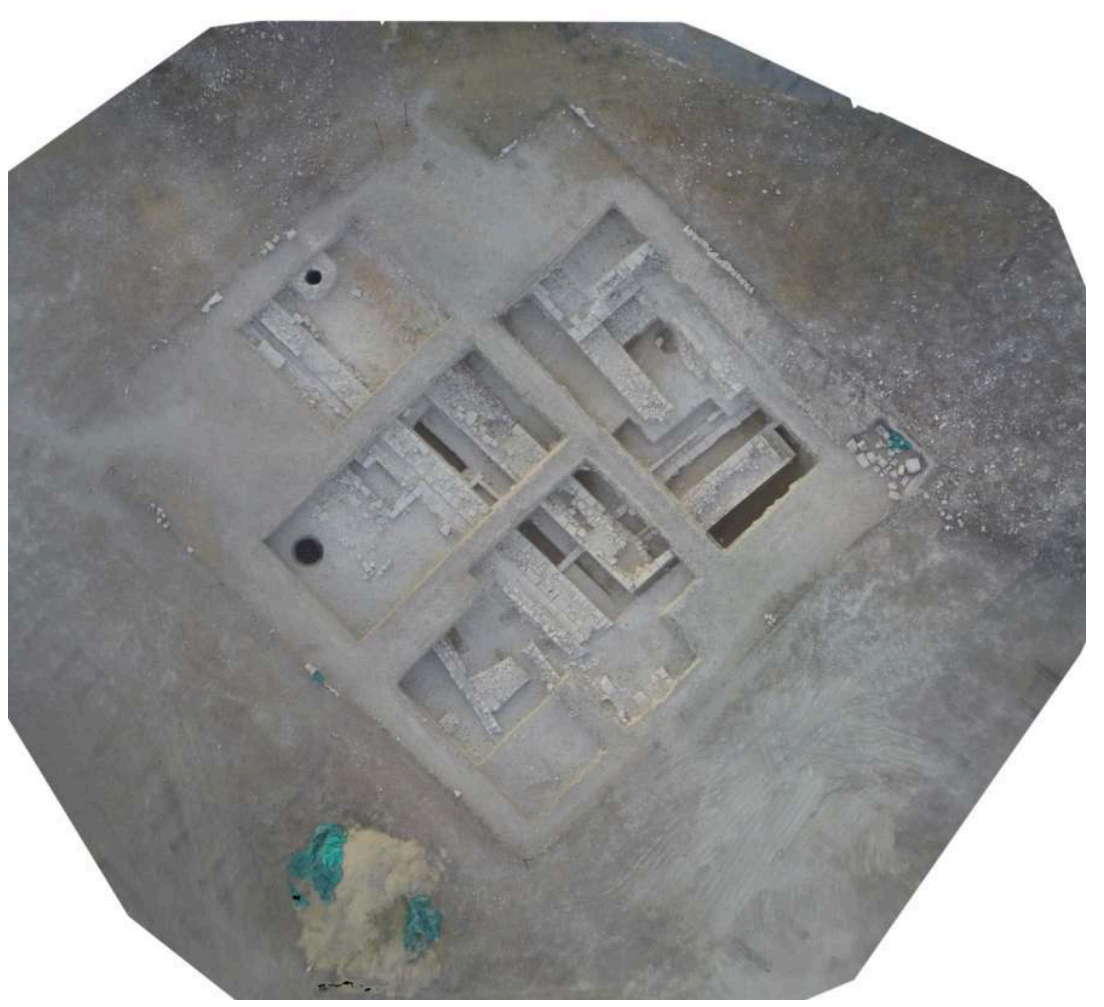

L. Thély / EFA. 


\section{UC 1001 et son démantèlement} imprégnée de pigments (remplissage US 2026, coupe US 2027) et de traces de feu confirme l'hypothèse formulée en 2015 de la présence d'activités artisanales liées au travail des pigments dans la partie Nord de la zone fouillée. Ces activités doivent, sans nul doute, être mises en rapport avec l'US «multicolore" recouvrant l'UC 1001 à l'Ouest qui présente des caractéristiques physiques semblables. En outre, le foyer US 2010 découvert en 2015 doit probablement être rattaché à la même phase (il contenait également de la culinaire présentant des traces de pigment). L'ensemble de ses activités se déploie sur des couches qui recouvrent l'UC 1001. On est donc en présence d'une seconde phase qui voit l'implantation d'activités artisanales (peut-être partiellement à ciel ouvert ou s'abritant dans des structures périssables) sur la zone.

\section{Le remblaiement et la construction des structures UC 2005,} UC 2002, UC 2006.

Le remblaiement de ces activités (US 2025) est à la base de la phase suivante. I semblerait qu'on ait rehaussé le terrain pour recouvrir et uniformiser le niveau de vie précédent. On a ensuite procédé à la construction d'UC 2006 puis 2005, avant d'ajouter un pan supplémentaire, UC 2002 (pour le détail, cf. infra). On assiste donc à une réappropriation de cet espace qui est à nouveau pourvu de construction, dont l'importance est pour l'instant impossible à déterminer en regard de l'étendue de la fenêtre ouverte par notre sondage.

\section{Spoliation et remblaiement d'UC 2005}

17 L'US 2119 semble nous indiquer que des blocs de la portion Est-Ouest d'UC 2005 (située au Sud) ont été prélevés. En effet, la trace laissée correspond à la largeur d'UC 2005 dans sa portion Nord-Sud (située à l'Est) : 1,35 m. L'espace laissé a ensuite été recouvert par de la terre mêlée à des résidus. Il se pourrait que la spoliation de la structure UC 2002 (il semble en effet qu'on n'en conserve que la fondation, cf. infra) soit intervenue durant la même période. La superposition d'US 2113 à US 2119 nous indique en outre que dans un second temps, l'espace interne a été remblayé. Il s'agit donc des traces de l'abandon de cette structure, qui constituent la dernière phase documentée avant les remaniements modernes de la zone. 


\section{Couches modernes (US 2101 à 2112 et 2114 à 2118)}

Les US 2101 à 2112 sont toutes postérieures aux strates 2114 à 2118 qui contenaient du matériel moderne. Il en résulte logiquement qu'elles sont elles aussi modernes. Il s'agit probablement de différentes phases de remaniement de l'espace dans un passé récent, peut-être à l'occasion de la construction de la route anglaise et ensuite du nettoyage annuel à la pelle mécanique de toute la zone au Sud de l'agora. Il semble donc que, jusqu'à une période relativement récente, impossible à dater plus précisément sans l'aide de documents d'archive, les structures UC 2005 et 2002 étaient encore visibles.

\section{Présentation choisie du matériel}

Quelques données concernant le matériel peuvent être présentées, mais devront être précisées à la suite des campagnes d'études.

La variété du matériel mis au jour reflète, en 2016 comme lors des campagnes précédentes, les phases de remblaiement antiques que nous avons fouillées: la céramique, si elle représente une part considérable des trouvailles, et dont la chronologie peut souvent être précisée à l'époque hellénistique et impériale, offre des faciès variés (culinaire africaine ou de production locale, céramique fine et commune ; enfin la moitié environ des tessons appartiennent à des amphores incomplètes).

1 La coroplastie chypriote est également bien représentée, ainsi que le verre, souvent à l'état fragmentaire.

2 Nous pouvons signaler quelques trouvailles remarquables pour cette campagne :

- Un fragment d'avant-bras d'une statue de Bès assez colossale, qui peut-être doit être rapproché de l'exemplaire conservé au musée de Limassol (AM 3800) ;

- Une petite tête en terre cuite sans doute d'époque hellénistique représentant un personnage usé par le temps et la fatigue dont les parallèles sont très peu nombreux, voire inexistants, et qui en ferait un hapax exceptionnel.

- Une soixantaine de lampes, essentiellement d'époque hellénistique, pour certaines dans un excellent état de conservation.

On notera enfin la découverte d'une amulette en faïence égyptienne représentant un dieu à tête de bélier, dont nous donnons ci-dessous un descriptif plus détaillé (fig. 6).

\section{Amulette d'un dieu à tête de bélier (AM 3764)}

$\mathrm{H}: 2,8 \mathrm{~cm} ; \mathrm{L}: 0,6 \mathrm{~cm} ; \mathrm{P}: 1 \mathrm{~cm}$.

Dominique Barcat

Amulette en faïence à la glaçure de couleur vert pâle. 
Fig. 6. Amulette égyptienne représentant un dieu à tête de bélier, AM 3764 .

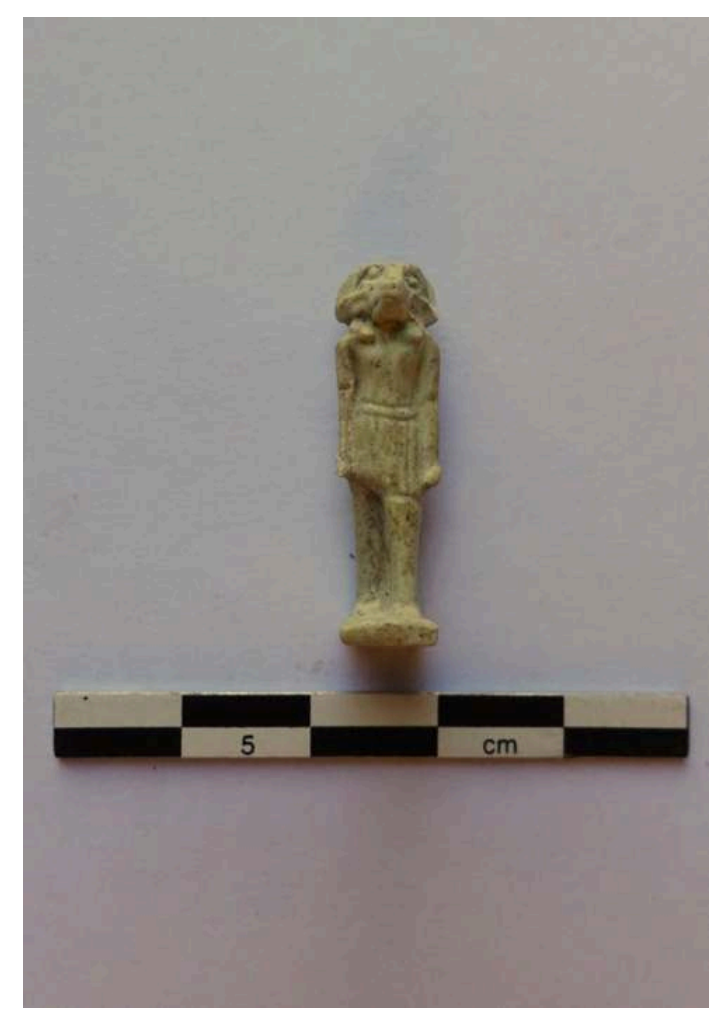

L. Thély / EFA.

24 Le dieu égyptien est représenté debout, sur une base rectangulaire, et adossé à un pilier, la jambe gauche en avant et les bras tendus le long du corps. Il porte la chenti (pagne rayé) et la perruque tripartite mais pas de couronne. Un trou de suspension est aménagé à l'arrière, à travers le pilier, à hauteur de la ceinture. On distingue clairement les cornes qui s'enroulent autour des tempes et se terminent au niveau des joues.

La bonne conservation de l'objet permet de juger d'une réalisation précise et soignée pour un objet de cette taille. On remarque néanmoins une exécution stylisée en particulier au niveau de la tête et des cornes. Cet exemplaire peut être comparé à une amulette du musée de Nicosie quasiment identique mais à qui la glaçure, plus épaisse, confère un aspect beaucoup plus imprécis ${ }^{6}$. Ici la glaçure vert pâle très fine rapporte cet objet à un ensemble d'amulettes produites en grand nombre en Égypte de la fin de la $\mathrm{XXVI}^{\mathrm{e}}$ dynastie jusqu'à l'époque hellénistique.

Les représentations d'un dieu à tête de bélier peuvent être rapportées à différentes divinités égyptiennes. Lorsque celui-ci est figuré sans le disque solaire ni l'urcus, il est d'usage de l'identifier au dieu Khnoum, le démiurge d'Éléphantine, voire à Hérishef, le dieu solaire d'Héracléopolis ${ }^{7}$. En principe la forme des cornes devrait permettre de distinguer Amon, qui les porte, comme ici, enroulées autour des tempes (tel le bélier de la race ovis platyura), de Khnoum, qui est traditionnellement doté de cornes torsadées s'étendant à l'horizontale de part et d'autre de sa tête comme celles de l'ovis longipes palaeo-aegyptiacus, aujourd'hui disparu. Cependant il est admis que la seconde forme est très rare sur les amulettes. Probablement pour des raisons pratiques qui concernent 
tant la fabrication que l'usage ${ }^{8}$, les cornes de l'ovis platyura se sont généralisées pour ce type d'objet.

L'identification de la divinité représentée n'a guère de sens hors de son contexte. Ainsi une amulette de dieu à tête de bélier découverte à Naucratis représente très probablement Amon-Baded, la divinité tutélaire de la ville, plutôt que Khnoum dont la présence à Naucratis n'est pas documentée ${ }^{9}$.

En Égypte, à partir de l'époque saïte, un certain nombre de momies sont parées d'une amulette de Khnoum, généralement placée sur la poitrine ${ }^{10}$ et, en règle générale, les figurines de dieu à tête de bélier découvertes ailleurs dans l'espace méditerranéen sont également issues de contextes funéraires. À Amathonte en particulier, ce sont les nécropoles qui ont livré la très grande majorité des amulettes de faïence recensées jusqu'à présent ${ }^{11}$. D'une de ces tombes amathousiennes provenait d'ailleurs un précédent exemplaire d'une amulette de dieu criocéphale ${ }^{12}$. Probablement, Khnoum, à la fois maitre de l'inondation et démiurge qui modèle le dieu-enfant sur son tour à partir du limon du Nil, est-il chargé d'une valeur fécondante et protectrice, notamment envers le jeune enfant. Sous l'Empire romain, le dieu apparaît d'ailleurs sur certaines gemmes magiques où il est représenté tenant la clé qui contrôle l'ouverture de l'utérus ${ }^{13}$. L'exemple des temple-boys, avec leurs multiples amulettes enfilées sur une même cordelette amène à penser que de telles amulettes en faïence étaient probablement portées par leur propriétaire de son vivant ${ }^{14}$.

Il faut cependant tenir compte du fait que quelques Aegyptiaca ont été découverts à Amathonte hors d'un contexte funéraire, et notamment dans le palais qui, situé sur l'acropole, surplombe l'emplacement où notre amulette a été découverte. Une zone de rejet installée dans les décombres du palais a ainsi livré un riche matériel votif de différentes époques, dont une amulette de la déesse Thouéris, une autre figure de fécondité, protectrice des femmes et des accouchements ${ }^{15}$. D'autre part P. Flourentzos a mis en évidence l'existence d'un dépôt situé à proximité de notre sondage, au pied de l'acropole dans la ville basse, dépôt qui a été interprété comme témoignant de l'existence d'un sanctuaire (bien qu'aucune fondation n'ait été dégagée) ${ }^{16}$. Le matériel à connotation égyptisante ainsi que la présence d'un autel dédié à Arsinoé II laisse supposer l'existence d'un sanctuaire dynastique ptolémaïque. Aucun objet de faïence n'a été relevé mais un œil oudjat en calcaire amène à penser que ce type d'objet aurait pu faire partie du mobilier. Il faut donc également envisager que ce dépôt puisse être à l'origine de notre amulette. Dans l'hypothèse où celle-ci proviendrait effectivement d'un sanctuaire, et a fortiori s'il s'agit d'un sanctuaire ptolémaïque, il faut avoir à l'esprit l'importante diffusion de la figure du dieu-bélier issue de l'Amon égyptien dans le contexte méditerranéen du ${ }^{\mathrm{er}}$ millénaire avant $\mathrm{n}$. è. À l'époque archaïque, le culte de Zeus-Ammon se répand depuis Cyrène et, quelques siècles plus tard, son oracle à Siwa se trouve au cœur du processus de légitimation d'Alexandre comme roi d'Égypte. Si Zeus-Ammon est généralement représenté avec un visage humain, ne conservant du bélier que les cornes, Chypre a produit à l'époque archaïque des représentations d'une divinité criocéphale très proche de l'iconographie égyptienne. C'est ce dont témoignent le monnayage produit sous Évelthon de Salamine (env. 560-525), ainsi que les statues et statuettes anthropomorphes d'un dieu criocéphale assis, probablement directement inspiré des représentations de l'Amon égyptien ${ }^{17}$, et dont un exemplaire en calcaire provient d'ailleurs d'Amathonte ${ }^{18}$. 
ne pouvons bien sûr rien affirmer en ce qui concerne le rôle et l'interprétation de cette amulette découverte hors de son contexte d'origine, néanmoins il est intéressant de voir que l'image du dieu criocéphale n'est pas étrangère à Amathonte.

\section{BIBLIOGRAPHIE}

ANDREWS 1994

Carol A.R. Andrews, Amulets of Ancient Egypt, Londres, Trustees of the British Museum, 1994.

BLANDIN, PETIT, TASSIGNON 2008

Béatrice Blandin, Thierry Petit, Isabelle Tassignon, « Recherches récentes au palais

d'Amathonte », CCEC 38, 2008, p. 129-142.

CLERC 1991

Gisèle Clerc, « Aegyptiaca », in Vassos Karageorghis, Olivier Picard, Christiane Tytgat (éd.), La

nécropole d'Amathonte, tombes 110-385: V, Nicosie-Athènes, A.G. Leventis Foundation et École

française d'Athènes, Études Chypriotes 13, 1991, p. 1-158.

CLERC 2014

Gisèle Clerc, « Aegyptiaca de la nécropole d'Agios Georgios », in Sophocles Hadjisavvas, The Phoenician Period Necropolis of Kition, II, Nicosie, Department of Antiquities, 2014, p. 56-134.

DASEN 2015

Véronique Dasen, Le sourire d'Omphale : maternité et petite enfance dans l'Antiquité, Rennes, Presses universitaires de Rennes, 2015.

EMPEREUR (éd.) 2018

Jean-Yves Empereur (éd.), The Hellenistic Harbour of Amathus: Underwater Excavations, 1984-1986. Volume 2: Artefacts Found during Excavations, Athènes, École française d'Athènes, Études Chypriotes 20, 2018.

EMPEREUR, KOŽELJ 2018

Jean-Yves Empereur, Tony Koželj, The Hellenistic Harbour of Amathus: Underwater Excavations, 1984-1986. Volume 1: Architecture and History, Athènes, École française d'Athènes, Études Chypriotes 19, 2018.

FLOURENTZOS 2007

Pavlos Flourentzos, «An unknown Graeco-Roman temple from the lower town of Amathous », CCEC 37, 2007, p. 299-306.

FOURRIER 2003

Sabine Fourrier, « Divinités égyptiennes à Chypre à l'époque archaïque », in Demetrios Michaelides, Vassiliki Kassianidou, Robert S. Merrillees (éd.), Egypt and Cyprus in Antiquity, Oxford, Oxbow, 2009, p. 97-103.

THÉLY 2015-2016

Ludovic Thély, «Inscriptions d'Amathonte XI. Un autel en l'honneur de Ptolémée X et Bérénice III découvert aux abords Sud-Ouest de l'agora ", BCH 139-140, 2015-2016, p. 463-484. 
THÉLY et al. 2015-2016

Ludovic Thély, Julien Adam, Camille Castres, Antoine Chabrol, François-Dominique Deltenre, Antigone Marangou, Cécile Rocheron, « Rapport sur les travaux de l'École française d'Athènes en 2014 et en 2015 : Amathonte. Les abords Sud-Ouest de l'agora », BCH 139-140, 2015-2016, p. 980-1016.

\section{NOTES}

1. Cf. l'étude géomorphologique entreprise par Antoine Chabrol et Pierre Stephan: Chronique des fouilles en ligne notice $\mathrm{n}^{\circ} 5067$, mise en ligne le 10 septembre 2015, consultée le 29 juin 2020, https://chronique.efa.gr/?kroute=report\&id=5067; Chronique des fouilles en ligne notice $\mathrm{n}^{\circ} 5427$, mise en ligne le 5 juillet 2016, consultée le 29 juin 2020, https://chronique.efa.gr/?kroute=report\&id=5427 ; THÉLY et al. 2015-2016, p. 984-987.

2. EMPEREUR, KOŽELJ 2018 ; EMPEREUR (éd.) 2018.

3. Chronique des fouilles en ligne notice $n^{0} 5067$, mise en ligne le 10 septembre 2015, consultée le 29 juin 2020, https://chronique.efa.gr/?kroute=report\&id=5067; THÉLY et al. 2015-2016, p. 984-987.

4. La dédicace ayant été gravée autour de 100-90 av. n. è., le remplissage du mur est donc postérieur : THÉLY 2015-2016.

5. À ce sujet, pour davantage de détails, cf. THÉLY et al. 2015-2016, p. 993-1001.

6. Amulette M 203.

7. ANDREWS 1994, p. 30.

8. ANDREWS 1994, p. 30.

9. British Museum, EA27535.

10. ANDREWS 1994, p. 31.

11. CLERC 1991.

12. British Museum, $1969,0401.128$ : Amathus, Site $D$, tomb 185 (750-300 av. n. è.); https://www.britishmuseum.org/collection/object/G_1969-0401-128. L'amulette est décrite comme "perforée à travers les cornes ", il semble donc qu'il s'agisse d'un type différent de la nôtre.

13. DASEN 2015, p. 130-131, fig. 4.9.

14. CLERC 2014, p. 111.

15. BLANDIN, PETIT, TASSIGNON 2008, p. 138, fig. 13.

16. FLOURENTZOS 2007, p. 299-306.

17. FOURRIER 2003, p. 97-103.

18. British Museum, $\mathrm{n}^{\circ} \mathrm{C} 223$. 


\section{INDEX}

\section{Thèmes : EFA}

chronologie https://ark.frantiq.fr/ark:/26678/pcrtpcAdBe2SFu

lieux https://ark.frantiq.fr/ark:/26678/pcrtrvSIfnFAUb

sujets https://ark.frantiq.fr/ark:/26678/pcrtwh5icnwutJ, https://ark.frantiq.fr/ark:/26678/

pcrt4HEc49rMp7, https://ark.frantiq.fr/ark:/26678/pcrtUtcfIvQKcB

Année de l'opération : 2016

\section{AUTEURS}

\section{LUDOVIC THÉLY}

Ministère de l'Europe et des Affaires étrangères

\section{JULIEN ADAM}

Université de Namur

DOMINIQUE BARCAT

Université de Fribourg

\section{CAMILLE CASTRES}

USR 3155 « Institut de recherche sur l'architecture antique (IRAA »

FRANÇOIS-DOMINIQUE DELTENRE

École française d'Athènes

ANTIGONE MARANGOU

Université de Rennes 2

\section{CÉCILE ROCHERON}

UMR 8546 « Archéologie et Philologie d'Orient et d'Occident (AOROC) » 